\title{
Padrão tecnológico em unidades de produção familiar de leite no Rio Grande do Sul relacionado com diferentes tipologias
}

\author{
Tecnologies the domestic dairy producers in Rio Grande do Sul relations among the distinctive tipologies
}

\author{
Saionara Araujo Wagner ${ }^{1}$ Ivaldo Gehlen ${ }^{2}$ José Maria Wiest $^{3}$
}

\section{RESUMO}

Os diferentes tipos de produtores familiares de leite, moderno convencionaL, em transição e tradicional, desenvolvem em suas unidades produtivas atividades diversificadas, o que lhes têm garantido a permanência na atividade, apesar da baixa remuneração da sua principal matéria-prima, o leite. A utilização de diferentes tipologias possibilitou a análise e avaliação de unidades produtivas envolvidas neste estudo, com o objetivo de identificar diferenças no padrão tecnológico, inserção no mercado, organização sistêmica da propriedade, identificação com a racionalidade de produção entre os diferentes tipos, dentro do mesmo estrato de produtores. A metodologia utilizada baseou-se na revisão de bibliografia, aplicação de questionário semi-estruturado e análise das variáveis que correspondem à problemática proposta. O produtor que está passando do tipo tradicional para o tipo moderno convencional, aqui denominado. Em transição, corresponde a $55 \%$ dos entrevistados, e é um produtor de faixa etária média de 35 anos, que vem realizando investimentos em máquinas e equipamentos de forma coletiva, fez investimentos em infra-estrutura na última década. diferentemente do tipo Moderno que investiu na atividade na década de 80 e do tipo Tradicional que não o fez. As principais conclusões produzidas por esta pesquisa são as de que a diversificação das atividades, presente em todos os tipos estudados, confere aos produtores de leite no Rio Grande do Sul, a permanência na atividade $e$ as transformações tecnológicas e de mercado os desafiaram a fazerem investimentos, na última década, a fim de aumentarem a produtividade e qualidade do leite, observação que pode ser confirmada pelo elevado número de produtores entrevistados classificados como do tipo em transição.

Palavras-chave: agricultura familiar, produção familiar de leite, sanidade animal, unidade de produção familiar.

\begin{abstract}
Dairy producers in small farms and different types (modern conventional, transitional and traditional) develop other activities besides milk, what can lead them to stay in the farm, due to the low income from milk. The use of the types of production allowed the analysis and evaluation of the farms involved in this study, with the aim of identifying differences in the technological patterns, market insertion, systemic organization and identification with the rationality of production among the different types, inside the same level of producers. The methodology was based on bibliography, the application of a semi-structured questionaire and the analysis of the variables that match the problematic proposed. The producers that are going from the traditional to the modern conventional type ( transitional ones, $55 \%$ of the interviewed farmers) are around 35 years old, invest in machinery and equipment as a group, did investments in substructure in the last decade, as opposed to the modern ones, that invested in the dairy activities in the 80's and traditional farmers, who did not invested so far. The main conclusion from this study shows that the diversion of activities - as presented by all types - allows the RS dairy producers to stay in the activity and that the technological and market advances were a challenge to investiments in the last decade with the aim of increasing production and quality of milk; this trend is shown by the number of interviewed farmers in the traditional stage.
\end{abstract}

Key words: small farms, family dairy production, animal health, family unit production.

\section{INTRODUÇÃO}

A presente pesquisa tem como objetivo avaliar os impactos e mudanças que os produtores

\footnotetext{
'Médico Veterinário, Doutor em Ciências Veterinária, Pesquisador da Fundação Estadual de Pesquisa Agropecuária (FEPAGRO), Rua Gonçalves Dias 570, 90130-060, Porto Alegre, RS. E.mail: saionara-wagner@ fepagro.rs.gov.br. Autor para correspondência. ${ }^{2}$ Sociólogo, Doutor em sociologia, Professor Adjunto, Departamento de Sociologia Rural, Instituto de Filosofia e Ciências Humanas (IFCH), Universidade Federal do Rio Grande do Sul (UFRGS).

${ }^{3}$ Médico Veterinário, Doutor em Ciências Veterinárias, Professor Adjunto, Departamento de Microbiologia de Alimento, Instituto de Ciência e Tecnologia de Alimentos (ICTA), UFRGS.
} 
familiares de leite enfrentaram principalmente na década de 90, com o advento das transformações tecnológicas e de mercado e da sua inserção na cadeia agroalimentar, processo desencadeador de modernização da atividade e ao mesmo tempo de exclusão de produtores.

O estudo está referenciado em 160 entrevistas semi-estruturadas com produtores familiares de leite das seguintes regiões: Vale do Taquari (Teutônia, Imigrante, Arroio do Meio, Paverama); Pelotas (Pelotas, São Lourenço do Sul); Serra (Nova Petrópolis), Noroeste (Três de Maio, Crissiumal, Boa Vista do Buricá, Selbach, Espumoso, Ibirubá); Metropolitana (Viamão e seu distrito de Itapuã). Os resultados das entrevistas aliados às observações dos pesquisadores nas propriedades possibilitaram a análise do sistema de produção nas diferentes tipologias de produtores familiares de leite.

O padrão de modernização, que passou pela organização técnicoprodutiva, ocorreu após intenso processo de transformação sócioeconômica pela qual passou a sociedade brasileira com o advento da industrialização e o progresso tecnológico alcançado. $\mathrm{O}$ que se observa na agricultura não é diferente do observado na indústria, ou seja, a modernização é definida pela mudança na base técnica de produção. Desta forma então, as técnicas tradicionais, baseadas na tração animal, uso de sementes próprias etc. são substituídas por implementos agrícolas modernos, sementes híbridas e insumos industriais (BRANDEMBURG 1999).

As mudanças de comportamento dos consumidores, a reestruturação da logística de industrialização e distribuição de lácteos, a abertura do mercado internacional determinaram uma rápida transformação na cadeia agro-industrial de leite no Brasil. Para sobreviver a essas mudanças ocorridas nos últimos anos, principalmente no que diz respeito às mudanças tecnológicas preconizadas, os produtores investiram em novas tecnologias e equipamentos a fim de atender a demanda das indústrias processadoras (ALEIXO, 2001; JANK e GALAN, 1999).

Na produção leiteira, o que se constatou foi que o padrão tecnológico moderno de produção gerou um processo de exclusão de 107.000 pequenos produtores familiares do mercado das oito maiores agroindústrias do setor, no período de 1996 e 2000, o que significou 56,21\% do número de produtores do seu mercado, conforme dados da Associação Brasileira dos Produtores de Leite (2002). Verificou-se, na presente pesquisa, que o processo de modernização dos produtores de leite exigido pelas agroindústrias, apresentou-se de forma diferente no Rio Grande do Sul. Aqui houve uma menor exclusão do número de produtores de leite que foi $26,85 \%$, conforme a mesma fonte. Isto se deve, em parte, ao fato de os produtores familiares de leite do Estado, em sua grande maioria, estarem organizados em cooperativas, o que garante a comercialização do leite mesmo em pequena escala.

Com base nessas informações é provável, que a menor exclusão desses produtores, também esteja relacionada ao fato dos diferentes tipos estudados, Moderno Convencional, Em Transição e Tradicional, independentemente do seu grau de tecnificação, serem produtores consolidados e terem atividades diversificadas em suas propriedades, proporcionandolhes outras fontes de renda o que revela o pluralismo dos produtores familiares de leite no Rio Grande do Sul.

\section{MATERIAL E MÉTODOS}

\section{Coleta de dados}

As entrevistas foram realizadas seguido os modelos de THIOLLENT (1996) e HAGUETTE(1990), aplicando-se um questionário semi-estruturado com visitação às propriedades. A pesquisa de campo foi realizada entre outubro e dezembro de 1999, com aplicação de 160 entrevistas com produtores familiares de leite, selecionados no Estado do Rio Grande do Sul. Foram coletados dados referentes a dois períodos,1990-94 e 1995-99, através de informação do próprio produtor entrevistado, a fim de que se pudesse avaliar a evolução da atividade leiteira nas unidades produtivas. A análise estatística baseou-se nos Teste de Qui-quadrado, análise de variância e teste de Tukey para comparação das análises e foi executada através do programa SPSS 8.0.

\section{Tipificação dos produtores de leite}

O uso de uma tipificação representativa do universo dos produtores familiares de leite se torna uma ferramenta importante, a fim de se adequar a um modelo inicial para uma pesquisa (WEBER, 2002).

Os Tipos Ideais foram construídos por Gehlen (2000), que coordenou uma pesquisa para estudar a questão da competitividade e identidade dos produtores familiares de leite no Estado do Rio Grande do Sul. Segundo o autor, os três Tipos Ideais constituem-se:

a) Produtor moderno convencional: produtor consolidado, ou seja, que apresenta um tempo de regularidade mínimo de cinco anos na atividade de forma comercial, identifica-se e tem uma racionalidade de produtor de leite moderno, sua produtividade está de acordo com o padrão moderno dentro da sua região. A produção de leite é estratégica na propriedade e utiliza a força de trabalho principal na atividade, sendo que essa se ocupa na sua maior parte na atividade leiteira. $\mathrm{Na}$ organização sistêmica da propriedade prioriza a atividade leiteira e o reinvestimento dos rendimentos se dá na sua maior parte na própria atividade. $\mathrm{O}$ padrão tecnológico 
adotado por esse produtor segue as especificações do pacote tecnológico ditado pelas agroindústrias, os animais são especializados na produção leiteira, a alimentação é balanceada e controlada de acordo com critérios técnicos especificados pela assistência técnica (geralmente prestada pelas grandes agroindústrias).

b) Produtor em transição: Também é um produtor consolidado, mas não se identifica completamente como produtor moderno e nem adota completamente esta racionalidade, sua produtividade não está de acordo com o padrão moderno dentro de sua região, pois sua média de produtividade é mais baixa. A produção de leite está se tornando estratégica e na organização sistêmica da propriedade, a força de trabalho principal está se envolvendo cada vez mais na atividade. O reinvestimento dos rendimentos se dá cada vez mais na atividade, embora ainda não seja a principal atividade. No que diz respeito ao padrão tecnológico, embora as instalações e os equipamentos ainda sejam um pouco precários, já houve aquisição de equipamentos adequados e construções específicas para a atividade. Os animais, embora na sua maioria sejam mestiços, já estão sendo melhorados geneticamente, e, em alguns casos, há aquisição de animais puros. A força de trabalho está se qualificando para a atividade, existe a percepção da necessidade de fazer um balanceamento adequado na alimentação dos animais e de prover alimento o ano inteiro, o que já começa a ser executado de forma gradual. Esse produtor está numa situação em que pode evoluir para um produtor Moderno Convencional, ou de acordo com a conjuntura, desistir da atividade.

c) Produtor tradicional: Também é um produtor consolidado. Identifica-se como tradicional e tem uma racionalidade compatível com a identidade de produtor tradicional, ou seja, sua produtividade está de acordo com o padrão tradicional na sua região. A produção de leite não é uma atividade estratégica dentro da propriedade, utilizando-se da força de trabalho secundária e apenas o tempo necessário, não sendo essa qualificada. Na organização sistêmica da propriedade, não prioriza a produção de leite, sendo que o reinvestimento dos rendimentos raramente se dá na atividade leiteira. No padrão tecnológico, as instalações e equipamentos, quando existem, são precários, os animais não são especializados, a alimentação não é balanceada e na maior parte do tempo é precária.

\section{RESULTADO E DISCUSSÃO}

Observou-se, no presente trabalho, que o produtor do tipo Moderno Convencional fez grandes investimentos na infra-estrutura de produção e compra de animais de raça, no final da década de 80 e início de 90 , contando com o auxílio de crédito externo e da assistência técnica prestada principalmente pela Cooperativa, na qual está associado. Atualmente este produtor não pensa em fazer novos investimentos, sua produção de leite nos últimos 15 anos (período estudado) está acima de 25.000 litros/ano, com uma produtividade entre 3.000 e 6.000 litros/vaca/ano, e um rebanho superior a 10 vacas. A atividade leiteira não é desenvolvida com exclusividade na unidade produtiva, sendo que as três principais fontes de renda são leite, cereais e gado de corte, conforme dados das tabelas 1,2 e 3 .

O produtor do tipo Em Transição investiu de forma mais acentuada nos últimos dez anos, tanto na infraestrutura de produção como na compra de animais de raça. A produção de leite passou de até 18.000 litros/ano para mais de 25.000 litros/ano neste período, com uma produtividade de até 3.000 litros/vaca/ano e um rebanho

Tabela 1 - Freqüência absoluta $(\mathrm{Fa})$ e relativa em \% (Fr) em relação à utilização de crédito e assistência técnica prestada pela cooperativa conforme tipologia do produtor nas diferentes categorias.

Tipo de produtor

\begin{tabular}{|c|c|c|c|c|c|c|}
\hline \multirow[t]{2}{*}{ Categorias } & \multicolumn{2}{|c|}{ Moderno convencional } & \multicolumn{2}{|c|}{ Em transição } & \multicolumn{2}{|c|}{ Tradicional } \\
\hline & $\mathrm{Fa}$ & Fr & $\mathrm{Fa}$ & $\mathrm{Fr}$ & $\mathrm{Fa}$ & Fr \\
\hline \multicolumn{7}{|c|}{ Utilização de crédito } \\
\hline Sempre & 22 & 40,0 & 25 & 33,8 & 6 & 24,0 \\
\hline No passado & 29 & 52,7 & 42 & 56,8 & 11 & 44,0 \\
\hline Nunca utilizou & 4 & 7,3 & 7 & 9,5 & 8 & 32,0 \\
\hline Total & 55 & 100,0 & 74 & 100,0 & 25 & 100,0 \\
\hline \multicolumn{7}{|c|}{ Assistência técnica } \\
\hline Sistematicamente & 38 & 65,5 & 22 & 30,1 & 7 & 25,0 \\
\hline De vez em quando & 9 & 15,5 & 17 & 23,3 & 15 & 53,6 \\
\hline Nunca & 11 & 19,0 & 34 & 46,6 & 6 & 21,4 \\
\hline Total & 58 & 100,0 & 73 & 100,0 & 28 & 100,0 \\
\hline
\end{tabular}


Tabela 2 - Freqüência absoluta (Fa) e relativa em \% (Fr) do número de vacas, da produção (litros/ano) e produtividade (litros/vaca/ano) de leite conforme tipologia do produtor nos períodos 1985 e 1999 nas diferentes categorias.

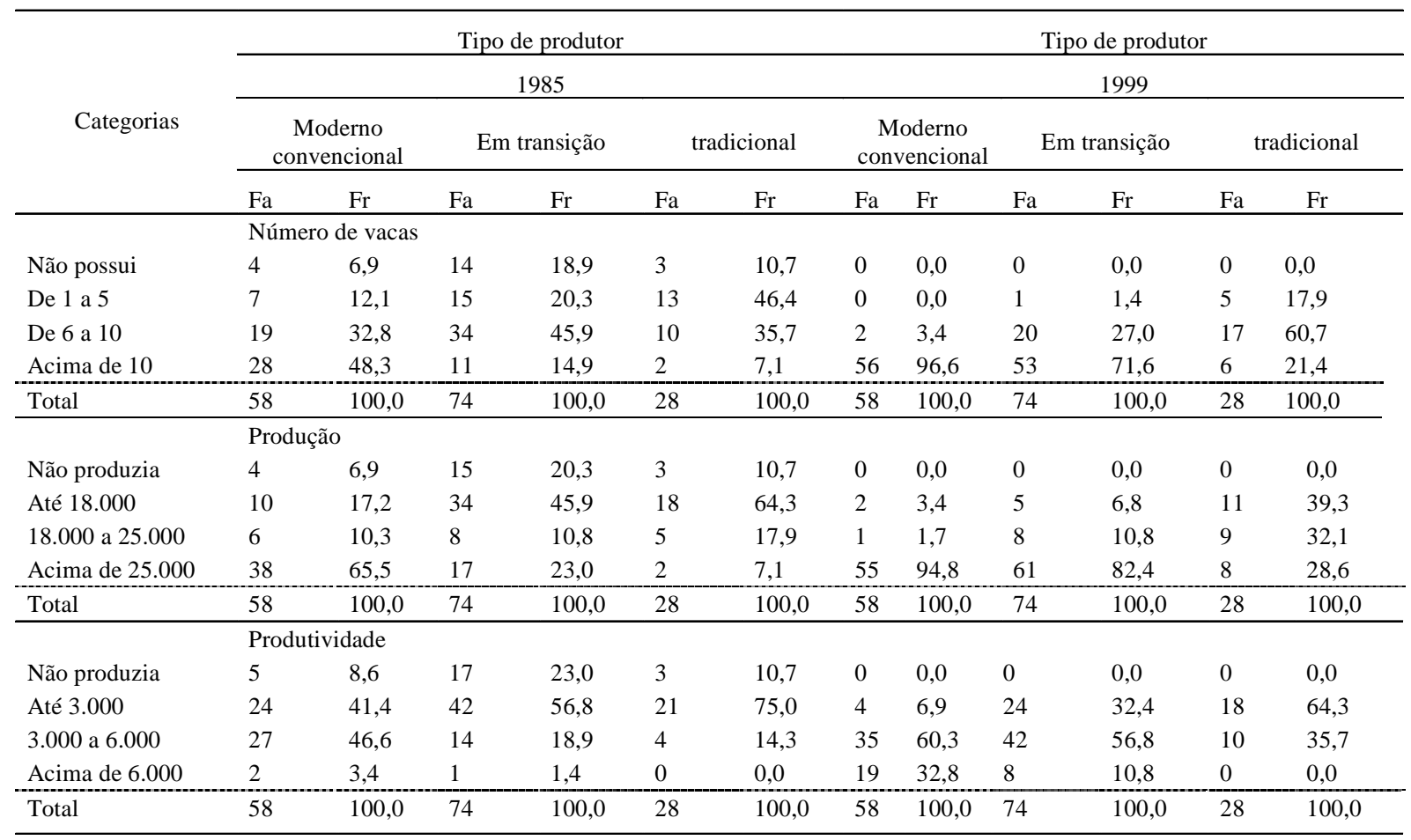

Tabela 3 - Freqüência absoluta (Fa) e relativa em \% (Fr) da utilização de máquinas e implementos conforme tipologia do produtor nas diferentes categorias.

\begin{tabular}{|c|c|c|c|c|c|c|c|c|c|c|c|c|}
\hline \multirow{3}{*}{ Categorias } & \multicolumn{6}{|c|}{ Tipo de produtor } & \multicolumn{6}{|c|}{ Tipo de produtor } \\
\hline & \multicolumn{2}{|c|}{$\begin{array}{c}\text { Moderno } \\
\text { convencional }\end{array}$} & \multicolumn{2}{|c|}{ Em transição } & \multicolumn{2}{|c|}{ Tradicional } & \multicolumn{2}{|c|}{$\begin{array}{c}\text { Moderno } \\
\text { convencional }\end{array}$} & \multicolumn{2}{|c|}{ Em transição } & \multicolumn{2}{|c|}{ Tradicional } \\
\hline & $\mathrm{Fa}$ & $\mathrm{Fr}$ & $\mathrm{Fa}$ & $\mathrm{Fr}$ & $\mathrm{Fa}$ & $\mathrm{Fr}$ & $\mathrm{Fa}$ & $\mathrm{Fr}$ & $\mathrm{Fa}$ & $\mathrm{Fr}$ & $\mathrm{Fa}$ & $\mathrm{Fr}$ \\
\hline & \multicolumn{6}{|c|}{ Trator } & \multicolumn{6}{|c|}{ Implementos trator } \\
\hline Não possui & 4 & 6,9 & 19 & 25,7 & 18 & 64,3 & 5 & 8,6 & 22 & 29,7 & 19 & 67,9 \\
\hline Individual & 45 & 77,6 & 38 & 51,4 & 6 & 21,4 & 47 & 81,0 & 36 & 48,6 & 5 & 17,9 \\
\hline Em grupo & 9 & 15,5 & 17 & 23,0 & 4 & 14,3 & 6 & 10,3 & 16 & 21,6 & 4 & 14,3 \\
\hline \multirow[t]{2}{*}{ Total } & 58 & 100,0 & 74 & 100,0 & 28 & 100,0 & 58 & 100,0 & 74 & 100,0 & 28 & 100,0 \\
\hline & \multicolumn{6}{|c|}{ Ordenhadeira } & \multicolumn{6}{|c|}{ resfriador } \\
\hline Não possui & 0 & 0,0 & 5 & 6,8 & 14 & 50,0 & 0 & 0,0 & 3 & 4,1 & 14 & 50,0 \\
\hline Individual & 56 & 96,6 & 64 & 86,5 & 14 & 50,0 & 56 & 96,6 & 64 & 86,5 & 13 & 46,4 \\
\hline Em grupo & 2 & 3,4 & 5 & 6,8 & 0 & 0,0 & 2 & 3,4 & 7 & 9,5 & 1 & 3,6 \\
\hline Total & 58 & 100,0 & 74 & 100,0 & 28 & 100,0 & 58 & 100,0 & 74 & 100,0 & 28 & 100,0 \\
\hline \multicolumn{7}{|c|}{ Ensiladeira } & \multicolumn{6}{|c|}{ sala de ordenha } \\
\hline Não possui & 17 & 29,3 & 31 & 41,9 & 24 & 85,7 & 28 & 48,3 & 48 & 64,9 & 25 & 89,3 \\
\hline Individual & 24 & 41,4 & 18 & 24,3 & 3 & 10,7 & 30 & 51,7 & 23 & 35,1 & 3 & 10,7 \\
\hline Em grupo & 17 & 29,3 & 25 & 33,8 & 1 & 3,6 & 0 & 0,0 & 0 & 0,0 & 0 & 0,0 \\
\hline \multirow[t]{2}{*}{ Total } & 58 & 100,0 & 74 & 100,0 & 28 & 100,0 & 58 & 100,0 & 74 & 100,0 & 28 & 100,0 \\
\hline & \multicolumn{6}{|c|}{ Ternereira } & \multicolumn{6}{|c|}{ Esterqueira } \\
\hline Não possui & 22 & 37,9 & 38 & 51,4 & 22 & 78,6 & 36 & 62,1 & 43 & 58,1 & 10 & 35,7 \\
\hline Individual & 36 & 62,1 & 36 & 48,6 & 6 & 21,4 & 22 & 37,9 & 31 & 41,9 & 18 & 64,3 \\
\hline Em grupo & 0 & 0,0 & 0 & 0,0 & 0 & 0,0 & 0 & 0,0 & 0 & 0,0 & 0 & 0,0 \\
\hline Total & 58 & 100,0 & 74 & 100,0 & 28 & 100,0 & 58 & 100,0 & 74 & 100,0 & 28 & 100,0 \\
\hline
\end{tabular}

Ciência Rural, v.34, n.5, set-out, 2004. 
oscilando entre 5 e 10 vacas, manifestando interesse em continuar investindo na atividade e aumentar a produção. Da mesma forma, a atividade leiteira não é desenvolvida com exclusividade na unidade produtiva, sendo que as três principais fontes de renda são leite, cereais e criação de suínos, aves e gado de corte. Para o tipo Tradicional observou-se, no período estudado, que esse continuou estagnado na atividade, tanto no que diz respeito à infraestrutura de produção, como em relação à produção de leite que permanece em até 18.000 litros/ano, com uma produtividade de até 3.000 litros/vaca/ano e um plantel de até 5 vacas, não manifestando interesse em aumentar a produção (Tabelas 2 e 3 ).

O manejo alimentare sanitário do plantel, quando observado através de diferentes tipologias, indica que o produtor do tipo Moderno Convencional organiza o sistema produtivo de forma a prover alimento diversificado e o ano todo aos animais. Essa alimentaçãoé composta de pastagem de inverno e verão, ração, silagem e sal mineral nos períodos estudados (1990-94 e 1995-99). O tipo Em Transição começa a organizar o sistema produtivo de forma a prover uma alimentação diversificada e o ano todo aos animais, somente no segundo período observado (1995-99). Observa-se também que os produtores do tipo Tradicional não organizaram o sistema produtivo de forma a prover alimento diversificado e o ano todo aos animais, embora demonstre utilizar com mais frequiência pastagem de inverno, no último período (Tabela4).

Em relação à infra-estrutura de produção da propriedade, observa-se que o produtor do tipo Moderno Convencional possui máquinas e equipamentos como: trator e seus implementos, ensiladeira, ordenhadeira, resfriador de leite suficientes para desenvolver a atividade leiteira de acordo com a sua capacidade de produção. $\mathrm{O}$ tipo Em Transição possui esses equipamentos de uma forma associativa, com exceção da ordenhadeira, mas tendendo a possuí-los de forma individual. Já o produtor do tipo tradicional não possui tais equipamentos. $\mathrm{O}$ mesmo acontece em relação às benfeitorias como sala de ordenha, esterqueira, ternereira (Tabela 3).

Quanto ao manejo sanitário do rebanho leiteiro, nas práticas como: controle de Tuberculose, vacinação para Brucelose, IBR, Leptospirose, observase que o produtor do tipo Moderno Convencional utiliza rotineiramente dessas práticas o que não ocorre com tipo Tradicional. Já o tipo Em Transição está começando a utilizar essas práticas como rotina (Tabela 5).

Tabela 4- Freqüência absoluta $(\mathrm{Fa})$ e relativa em \% (Fr) quanto à utilização de pastagem de inverno, de pastagem de verão, de silagem, de ração e de sal mineral conforme tipologia do produtor nos períodos de 1990 a 1994 e 1995 a 1999 nas diferentes categorias.

\begin{tabular}{|c|c|c|c|c|c|c|c|c|c|c|c|c|}
\hline \multirow{4}{*}{ Categorias } & \multicolumn{6}{|c|}{ Tipo de produtor } & \multicolumn{6}{|c|}{ Tipo de produtor } \\
\hline & \multicolumn{6}{|c|}{1990 a 1995} & \multicolumn{6}{|c|}{1995 a 1999} \\
\hline & \multicolumn{2}{|c|}{$\begin{array}{c}\text { Moderno } \\
\text { convencional }\end{array}$} & \multicolumn{2}{|c|}{ Em transição } & \multicolumn{2}{|c|}{ Tradicional } & \multicolumn{2}{|c|}{$\begin{array}{c}\text { Moderno } \\
\text { convencional }\end{array}$} & \multicolumn{2}{|c|}{ Em transição } & \multicolumn{2}{|c|}{ Tradicional } \\
\hline & $\mathrm{Fa}$ & $\mathrm{Fr}$ & $\mathrm{Fa}$ & $\mathrm{Fr}$ & $\mathrm{Fa}$ & $\mathrm{Fr}$ & $\mathrm{Fa}$ & $\mathrm{Fr}$ & $\mathrm{Fa}$ & $\mathrm{Fr}$ & $\mathrm{Fa}$ & $\mathrm{Fr}$ \\
\hline & \multicolumn{12}{|c|}{ Pastagem de inverno } \\
\hline Sim & 50 & 86,2 & 54 & 73,0 & 18 & 64,3 & 58 & 100,0 & 72 & 97,3 & 22 & 78,6 \\
\hline Não & 8 & 13,8 & 20 & 27,0 & 10 & 35,7 & 0 & 0,0 & 2 & 2,7 & 6 & 21,4 \\
\hline \multirow[t]{2}{*}{ Total } & 58 & 100,0 & 74 & 100,0 & 28 & 100,0 & 58 & 100,0 & 74 & 100,0 & 28 & 100,0 \\
\hline & \multicolumn{12}{|c|}{ pastagem de verão } \\
\hline Sim & 44 & 75,9 & 37 & 50,0 & 15 & 53,6 & 48 & 82,8 & 50 & 67,6 & 17 & 60,7 \\
\hline Não & 14 & 24,1 & 37 & 50,0 & 13 & 46,4 & 10 & 17,2 & 24 & 32,4 & 11 & 39,3 \\
\hline \multirow[t]{2}{*}{ Total } & 58 & 100,0 & 74 & 100,0 & 28 & 100,0 & 58 & 100,0 & 74 & 100,0 & 28 & 100,0 \\
\hline & \multicolumn{12}{|c|}{ Silagem } \\
\hline Sim & 51 & 87,9 & 43 & 58,1 & 9 & 32,1 & 55 & 94,8 & 63 & 85,1 & 15 & 53,6 \\
\hline Não & 7 & 12,1 & 31 & 41,9 & 19 & 67,9 & 3 & 5,2 & 11 & 14,9 & 13 & 46,4 \\
\hline \multirow[t]{2}{*}{ Total } & 58 & 100,0 & 74 & 100,0 & 28 & 100,0 & 58 & 100,0 & 74 & 100,0 & 28 & 100,0 \\
\hline & \multicolumn{12}{|c|}{ ração } \\
\hline Sim & 49 & 84,5 & 43 & 58,1 & 18 & 64,3 & 54 & 93,1 & 54 & 73,0 & 21 & 75,0 \\
\hline Não & 9 & 15,5 & 31 & 41,9 & 10 & 35,7 & 4 & 6,9 & 20 & 27,0 & 7 & 25,0 \\
\hline \multirow[t]{2}{*}{ Total } & 58 & 100,0 & 74 & 100,0 & 28 & 100,0 & 58 & 100,0 & 74 & 100,0 & 28 & 100,0 \\
\hline & \multicolumn{12}{|c|}{ Sal mineral } \\
\hline Sim & 53 & 91,4 & 56 & 75,5 & 25 & 89,3 & 57 & 98,3 & 63 & 85,1 & 26 & 92,9 \\
\hline Não & 5 & 8,6 & 18 & 24,3 & 3 & 10,7 & 1 & 1,7 & 11 & 14,9 & 2 & 7,1 \\
\hline Total & 58 & 100,0 & 74 & 100,0 & 28 & 100,0 & 58 & 100,0 & 74 & 100,0 & 28 & 100,0 \\
\hline
\end{tabular}

Ciência Rural, v.34, n.5, set-out, 2004. 
Tabela 5 - Freqüência absoluta $(\mathrm{Fa})$ e relativa em \% (Fr) da utilização das práticas sanitárias conforme tipologia do produtor nas diferentes categorias.

\begin{tabular}{|c|c|c|c|c|c|c|c|c|c|c|c|c|}
\hline \multirow{3}{*}{ Categorias } & \multicolumn{6}{|c|}{ Tipo de produtor } & \multicolumn{6}{|c|}{ Tipo de produtor } \\
\hline & \multicolumn{2}{|c|}{$\begin{array}{c}\text { Moderno } \\
\text { convencional }\end{array}$} & \multicolumn{2}{|c|}{ Em transição } & \multicolumn{2}{|c|}{ tradicional } & \multicolumn{2}{|c|}{$\begin{array}{c}\text { Moderno } \\
\text { convencional }\end{array}$} & \multicolumn{2}{|c|}{ Em transição } & \multicolumn{2}{|c|}{ Tradicional } \\
\hline & $\mathrm{Fa}$ & $\mathrm{Fr}$ & $\mathrm{Fa}$ & $\mathrm{Fr}$ & $\mathrm{Fa}$ & $\mathrm{Fr}$ & $\mathrm{Fa}$ & $\mathrm{Fr}$ & $\mathrm{Fa}$ & $\mathrm{Fr}$ & $\mathrm{Fa}$ & $\mathrm{Fr}$ \\
\hline & \multicolumn{6}{|c|}{ vacina brucelose } & \multicolumn{6}{|c|}{ vacina carbúnculo sintomático } \\
\hline Sim & 45 & 77,6 & 43 & 58,1 & 10 & 35,7 & 34 & 58,6 & 34 & 45,9 & 9 & 32,1 \\
\hline Não & 13 & 22,4 & 31 & 41,9 & 18 & 64,3 & 24 & 41,4 & 40 & 54,1 & 19 & 67,9 \\
\hline \multirow[t]{2}{*}{ Total } & 58 & 100,0 & 74 & 100,0 & 28 & 100,0 & 58 & 100,0 & 74 & 100,0 & 28 & 100,0 \\
\hline & \multicolumn{6}{|c|}{ vacina leptospirose } & \multicolumn{6}{|c|}{ teste tuberculose } \\
\hline Sim & 27 & 46,6 & 19 & 25,7 & 3 & 10,7 & 44 & 75,9 & 43 & 58,1 & 9 & 32,1 \\
\hline Não & 31 & 53,4 & 55 & 74,3 & 25 & 89,3 & 14 & 24,1 & 31 & 41,9 & 19 & 67,9 \\
\hline \multirow[t]{2}{*}{ Total } & 58 & 100,0 & 74 & 100,0 & 28 & 100,0 & 58 & 100,0 & 74 & 100,0 & 28 & 100,0 \\
\hline & \multicolumn{6}{|c|}{ vacina IBR } & \multicolumn{6}{|c|}{ vacina carbúnculo hemático } \\
\hline Sim & 23 & 39,7 & 15 & 20,3 & 0 & 0,0 & 31 & 53,4 & 34 & 45,9 & 7 & 25,0 \\
\hline Não & 35 & 60,3 & 59 & 79,7 & 28 & 100,0 & 27 & 46,6 & 40 & 54,1 & 21 & 75,0 \\
\hline Total & 58 & 100,0 & 74 & 100,0 & 28 & 100,0 & 58 & 100,0 & 74 & 100,0 & 28 & 100,0 \\
\hline
\end{tabular}

\section{CONCLUSÃO}

A diversificação das fontes de renda, através do desenvolvimento das diferentes culturas e criações, presente nas três Tipologias de produtores leiteiros estudadas, lhes confere a permanência na atividade leiteira e reafirma a agricultura de base familiar como um modelo sustentável de produção, independente da tecnologia adotada pelos mesmos.

\section{REFERÊNCIAS BIBLIOGRÁFICAS}

ALEIXO, S.S. et al. A importância das características sociais na elaboração de estratégias de desenvolvimento para a pecuária leiteira. In: CONGRESSO BRASILEIRO DE ECONOMIA E SOCIOLOGIA RURAL, 49., 2002, Passo Fundo. Anais... Brasília : SOBER : UPF, 2002. Cd-rom: il.
ASSOCIAÇÃO BRASILEIRA DE PRODUTORES DE LEITE. Maiores empresas de laticínios do Brasil. 2002. Disponível na internet: http://www.leitebrasil.org.br. On line. Acesso em novembro de 2002.

RANDEMBURG, A. Colonos: subserviência e autonomia. Para pensar outra Agricultura. Curitiba : UFPR, 1998. p.71-102.

GEHLEN, Ivaldo. Identidade e competitividade dos produtores familiares de leite/RS. Porto Alegre, 2000. (mimeo).

HAGUETTE, T.M. Metodologias qualitativas na sociologia. 2.ed. Petrópolis: Vozes, 1990. 163p.

JANK, M.; GALAN, V. O agrobusiness do leite no Brasil. São Paulo : Milkbizz, 1999. 28p.

THIOllent, M. Metodologia da pesquisa-ação. São Paulo : Cortez, 1996. 108p.

WEBER, M. Metodologia das ciências sociais. Parte 1. Campinas : Unicamp. 1992. 210p. 